Paediatr.Paedolog.2020 55 (Suppl 3):S144-S148 https://doi.org/10.1007/s00608-020-00771-8

(c) Der/die Autor(en) 2020

\section{Wolfgang Sperl}

Zentrum für Kinder- und Jugendmedizin Universitätsklinik für Kinder- und Jugendheilkunde, Paracelsus Medizinische Privatuniversität (PMU) Uniklinikum Salzburg - Landeskrankenhaus, Salzburg, Österreich

\title{
Kinder- und Jugendrehabilitation in Österreich: aktueller Stand
}

Nachdem jahrzehntelang die Kinderund Jugendrehabilitation in Österreich ungeregelt war, was sowohl die Finanzierung, als auch das Angebot betraf (zahlreiche Erwachsenenrehabilitationsbetten standen wenigen Kinderrehabilitationsbetten an Erwachseneneinrichtungen gegenüber), ist in den letzten 15 Jahren doch eine kontinuierliche Entwicklung in Richtung einer umfassenden österreichweiten Versorgung passiert.

\section{Ausgangspunkte und Durchbrüche}

Ein wesentlicher Ausgangspunkt war der Österreichische Gesundheitsplan für Kinder 2004, wo die verschiedenen Rehabilitationsformen im Kinder- und Jugendlichenbereich definiert wurden, von der Frührehabilitation über die Phase 3 Rehabilitation bis hin zur ambulanten Rehabilitation. Ein nächster Meilenstein war der Kindergesundheitsdialog 2010, wo eine eigene Arbeitsgruppe 4 für das Thema Rehabilitation für Kinder und Jugendliche eingerichtet wurde. Letztlich wurde von Gesundheit Österreich GmbH (GÖG) zusammen mit der Österreichischen Gesellschaft für Kinderund Jugendheilkunde (ÖGKJ) Indikationsgruppen und Bettenbedarfszahlen ermittelt [2]. Der Abschluss der Arbeitsgruppe 4 hatte auch ein Leistungsprofil für die stationäre Rehabilitation für Kinder und Jugendliche zum Inhalt. Letztlich wurde im Österreichischen Strukturplan für Gesundheit (ÖSG) 2012 der Kinderrehabilitationsplan mit entsprechender Bettenaufteilung und Zuteilung zu den Gesundheitsversorgungszonen aufgenommen.

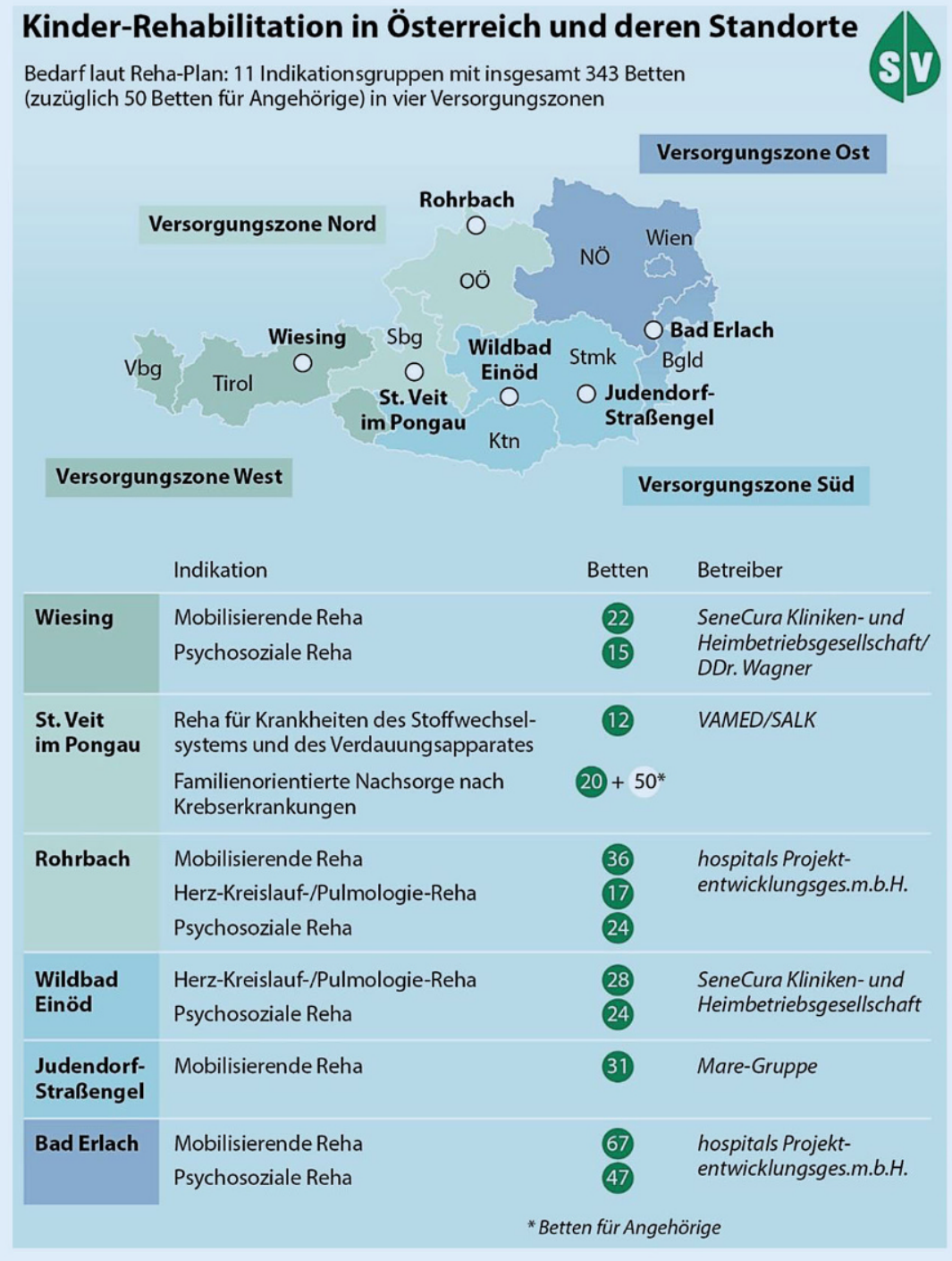

Abb. $1 \Delta$ Kinder- und Jugend Rehabilitationseinrichtungen in Österreich mit Indikationen und Betten. (Quelle: Homepage Sozialversicherungen, $[3,4]$ ) 

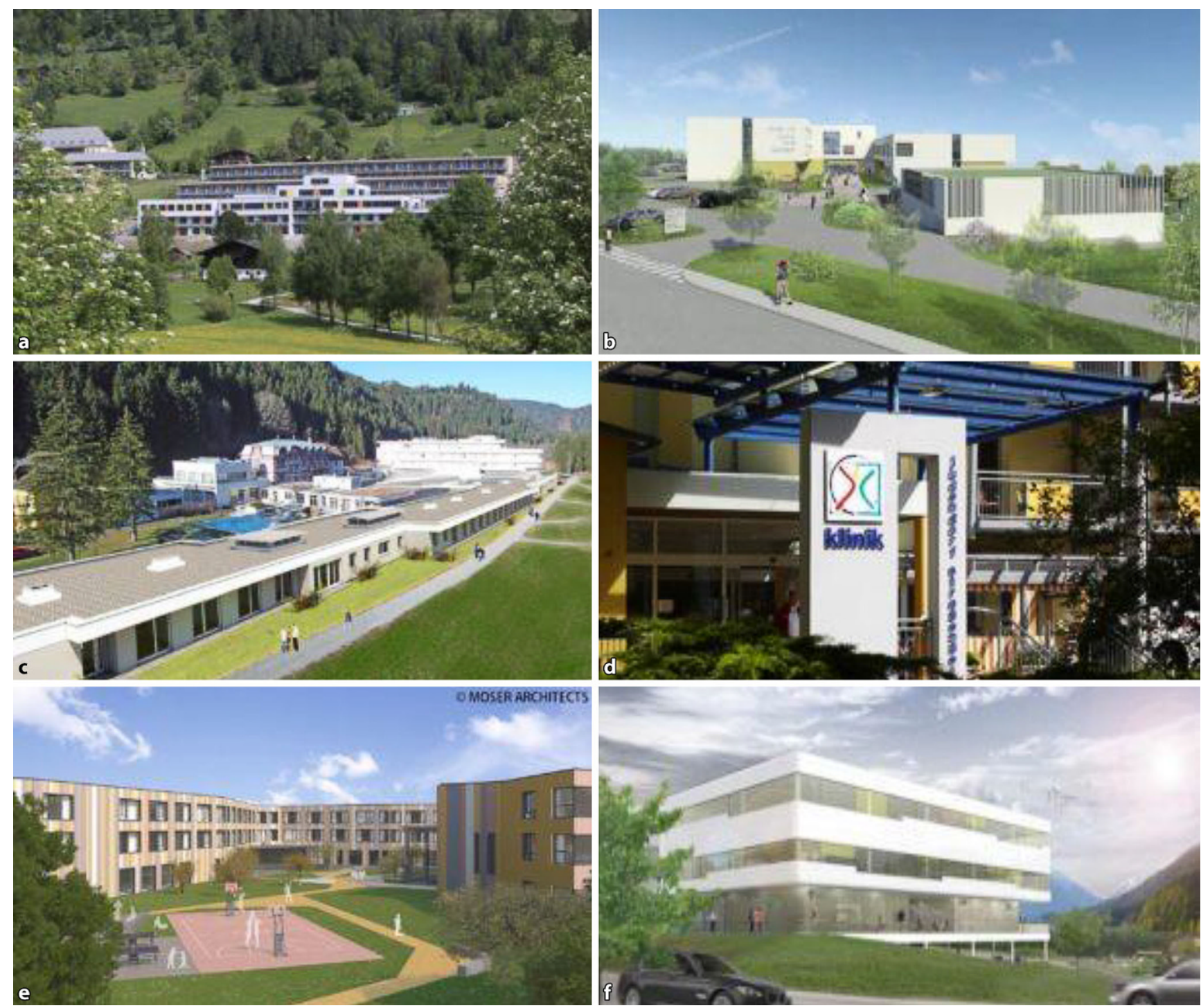

Abb. $2 \Delta$ Kinder- und Jugend Rehabilitationseinrichtungen in Österreich. a St. Veit im Pongau VAMED/SALK (Salzburger Landeskliniken, Salzburg), b Rohrbach Hospitals (Oberösterreich), c Wildbad Einöd Senecura (Steiermark), d Judendorf-Straßengel Mare-Gruppe (Steiermark), e Bad Erlach Hospitals (Niederösterreich), f Wiesing SeneCura/Dr.Dr.Wagner, Tirol (@ Förderverein Kinderreha [5])

Ein wesentlicher Durchbruch war auch 2015 die Bund-Länder-Einigung bezüglich der Finanzierung der Kinder- und Jugendrehabilitation. Immerhin spielen zwei Teile hier herein: die angeborenen - und die erworbenen Störungen. Schließlich wurde mit dem zur Verfügung stehenden Geld von 33 Mio. $€$ mit dem ermittelten Bettenbedarf von 325 Betten ein Ausschreibungsverfahren durch den Hauptverband für die $4 \mathrm{Ge}$ sundheitsregionen durchgeführt. Das Vergabeverfahren sah vor, gleichzeitig für ganz Österreich ein Konzept umzusetzen. Projekte, die bereits jahrelang in der Warteschleife waren, wurden damit an die Startlinie zurückgestellt. Die Ausschreibung war 2017 abgeschlossen und somit wurde der Beginn für die Umsetzung in den jeweiligen Gesundheitsregionen für die verschiedenen Betreiber ermöglicht ([1]; - Abb. 1 und 2: Kinderund Jugendrehabilitationseinrichtungen in den jeweiligen Gesundheitsregionen nach dem offiziellen Vergabeverfahren durch den Hauptverband). Dann wurden die ersten Einrichtungen in den verschiedenen Behandlungsindikationen in Judendorf Straßengel und St. Veit im Pongau im Leuwaldhof 2018 in Angriff genommen; 2019 wurden weitere Einrichtungen wie Bad Erlach und Rohrbach eröffnet. Ausstehend ist schließlich noch der Standort Wiesing (Mobilisierungsbzw. psychosoziale Rehabilitation).

Die ÖGKJ mit dem Referat für Rehabilitation hat in Zusammenarbeit mit dem Hauptverband der Sozialversicherungsträger Rehabilitationsvernetzungstreffen organisiert; 2 Referatssitzungen der Kinder- und Jugendrehabilitation wurden jeweils im Anschluss an die Jahrestagungen der ÖGKJ 2018 in Linz und 2019 in Klagenfurt abgehalten. 


\section{Positiver Rückblick}

Wenn man nun die Erfahrungen der ersten beiden Jahre der Kinder- und Jugendrehabilitation in Österreich betrachtet, dann ist es klar festzustellen, dass die jeweiligen Betreiber sehr engagierte Teams mit kompetenten Fachkräften sowohl im ärztlichen als auch im pflegerischen und therapeutischen Bereich gewonnen haben.

Es gibt von den Familien, die bereits in den Rehabilitationseinrichtungen waren, eindeutig erste sehr erfreuliche und positive Rückmeldungen. Es ist auch ersichtlich, dass sich die Rehabilitationseinrichtungen untereinander abstimmen, die jeweiligen ärztlichen und therapeutischen Vertreter die Kontakte untereinander suchen. Die Arbeitsgruppen der ÖGKJ mit den verschiedenen Subspezialisierungen sind aktiv geworden und bemühen sich für Gesamtkonzepte in der Versorgung (wie z. B. in der Adipositas, dem Diabetes, aber auch in der Nephrologie), die in die spezifische Rehabilitation eingebunden sind. Die Träger sind engagiert und haben kind- und jugendgerecht mit den Einrichtungen gestartet. Auch Selbsthilfegruppen sind involviert, insbesondere in der Kinderkardiologie das „Kinderherz“, aber auch bei der familienorientierten Rehabilitation im hämatoonkologischen Bereich die Österreichische Kinderkrebshilfe.

Ohne die Initiative für Kinder- und Jugendrehabilitation durch Markus Wieser, der auch teilweise den Außenauftritt und die Koordination der Einrichtungen übernommen hat und auch immer wieder Spendengelder für zusätzliche Unterstützungsleistungen für Familien zur Verfügung stellt und auch vom Hauptverband aus beauftragte Qualitätssicherungsmaßnahmen aus Sicht der Familien und Betroffenen durchführt, wäre die gesamte Entwicklung und der gute Start nicht möglich gewesen. Für den Leuwaldhof in St. Veit im Pongau wurde eine spezielle Qualitätssicherung und Datenakquise vorbildlich eingerichtet.

\section{Kritische Aspekte}

Neben diesen positiven Punkten gibt es auch einige kritische Aspekte: Es war

Paediatr. Paedolog. 2020 · 55 (Suppl 3):S144-S148 https://doi.org/10.1007/s00608-020-00771-8 (c) Der/die Autor(en) 2020

\section{W. Sperl}

\section{Kinder- und Jugendrehabilitation in Österreich: aktueller Stand}

\section{Zusammenfassung}

Nach jahrelangen Vorarbeiten wurde nach Erstellung eines Rehabilitationsplans für Kinder und Jugendliche, der Betten- und Indikationszuordnungen zu die jeweiligen Gesundheitsregionen und auch der Klärung der Finanzierung der Tagessätze nun die Errichtung von Kinder- und Jugendlichenrehabilitationszentren in Österreich durchgeführt. Der Start ist gelungen, engagierte MitarbeiterInnen konnten in allen Berufsbereichen für eine kinder- und jugendlichensspezifische Rehabilitation gewonnen werden. Kritische Punkte sind die Mitaufnahme von Begleitpersonen bzw. anderer Familienangehöriger, wobei eine Z-Diagnose für Begleitpersonen analog der familienorientierten Rehabilitation hämatoonkologischer Kinder für alle Indikationen wichtig wäre, um die entsprechende Freistellung vom Arbeitgeber zu bekommen. Ein wesentlicher Punkt ist auch die fallweise Ausweitung der familienorientierten Rehabilitation über die Hämatoonkologie hinaus. Die Transition sollte ebenfalls geregelt werden, gerade an der Schnittstelle zum
Erwachsenenalter gibt es Überlappungsbereiche wie z.B. bei Stoffwechselindikationen oder Hämatoonkologie. Wesentlich ist eine optimale und akkordierte Gestaltung der kinder- und jugendpsychiatrischen Rehabilitation in Absprache mit der kinderund jugendpsychiatrischen Fachgesellschaft. Wichtig ist außerdem, die Rehabilitation der jeweiligen Indikationsgruppe in die Gesamtversorgungskonzepte einzubauen, dieser Prozess ist derzeit im Gange. Zuletzt ist eine abgestimmte Qualitätssicherung aller Rehabilitationseinrichtungen sinnvoll. Die Entwicklung einer entsprechenden Rehabilitationskultur ist wichtig, ebenso die enge Zusammenarbeit zwischen Rehabilitationszentren und Zuweisern, um das vorhandene Potential optimal nutzen zu können.

Schlüsselwörter Rehabilitation · Kinder und Jugendliche . Familienorientierte Rehabilitation . Versorgungszonen · Pädiatrie

\section{Rehabilitation of Children and Adolescents in Austria: Actual Situation}

\section{Abstract}

After many years of preparation, children's and adolescents' rehabilitation centers in Austria have now been established according to the creation of a rehabilitation plan for children and adolescents, to the allocation of beds and indications to each health care region, and also to clarification regarding the payment of the daily rates. Dedicated staff have been recruited in all job sectors of rehabilitation for children and adolescents, resulting in a successful start. A critical point is the admission of chaperones or other family members whereby a $Z$ code diagnosis analogous to the family-oriented rehabilitation of hemato-oncological children would be important for all indications, so that they can take the relevant leave of absence from their employment. It is also important to widen the concept of familyoriented rehabilitation beyond the field of hemato-oncology. In addition, transition has to be regulated, as there is considerable overlap with adult patients in fields such as metabolic diseases and hemato-oncology. Optimal and synchronized planning of the psychiatric rehabilitation of children and adolescents in consultation with the Austrian Society of Child and Adolescent Psychiatry is crucial. Furthermore, it is important to integrate rehabilitation of each of the clinical diagnostic groups into the overall concept of care and this process is currently underway. Finally, coordinated quality assurance for the rehabilitation centers is judicious. The development of an appropriate rehabilitation culture is important, as is close cooperation between rehabilitation units and referring clinicians in order to make the best use of existing potential.

\section{Keywords}

Rehabilitation - Children and adolescents . Family-oriented rehabilitation - Health care regions . Pediatrics 
ersichtlich, dass sich beim fast gleichzeitigen Start aller Einrichtungen und dem Prinzip des Hochfahrens der Reha Probleme von $0 \%$ auf $100 \%$ entwickeln können: Das Personal Recruiting ist im Fachbereich Kinder- und Jugendheilkunde nicht einfach, sowohl was die medizinische Versorgung, die Ärztinnen und Ärzte, angeht, aber auch die Pflegeund andere Spezialkräfte. Durch die Zusammenfassung von Entwicklungs- und Sozialpädiatrie und Kinder- und Jugendpsychiatrie in Mental Health, durch die Verortung kinder- und jugendpsychiatrischer Versorgung im Mental-health-Bereich sind in der Entwicklung und in der Kommunikation besonders die Österreichische Gesellschaft für Kinder- und Jugendpsychiatrie nicht ausreichend involviert gewesen. Hier gilt es, in starker $\mathrm{Zu}$ sammenarbeit die vorhandenen Ressourcen optimal zu nutzen und Konzepte zu entwickeln. Einzelne Bereiche wie die Rehabilitation der nephrologischen Patienten sind nicht ausreichend berücksichtigt worden. Bei der Mobilisierungsindikation in der Phase C gibt es Qualitätsprobleme im Orthopädiekonzept, im Bereich der hämotoonkologischen familienorientierten Orientierung braucht es die Expertise, die neuropsychologische Versorgung, die familienorientierten Konzepte, die teilweise auch im Ausland in der Katharinenhöhe und in Tannheim praktiziert werden. Naturgemäß ist bei gleichzeitigem Eröffnen der vielen Betten eine gewisse Konkurrenzierung um die Patienten gegeben. Es gehört klar geklärt, welche Patienten welcher Indikationsgruppe wohin zugewiesen werden müssen. Hier ist der Überlappungsbereich für psychosomatische Versorgung gerade im Bereich Diabetes, Adipositas und anderen Krankheiten entsprechend wie es im Bereich der Erwachsenenrehabilitation erfolgt, zu klären. Der Standort Wiesing ist noch nicht ganz umgesetzt und hat eine neue Lokation erfahren, hier benötigt es erst die Vernetzung und Entwicklung am Standort.

\section{To-Do-Liste}

Aus der Referatssitzung der ÖGKJ sind zusammenfassend folgende Forderungen erstellt worden:

1. Eine Broschüre sollte erstellt werden mit der Bekanntmachung der Rehabilitationseinrichtungen und Indikationen in Österreich (gegebenenfalls durch die ÖGKJ und den Hauptverband bzw. Österreichische Gesundheitskasse [ÖGK]).

2. Es sollte 2020 wiederum ein Vernetzungstag erfolgen, nachdem alle Rehabilitationseinrichtungen gestartet sind, und dort ein Austausch erfolgen und eine Zwischenbilanz gezogen werden.

3. Ganz wesentlich erscheint es, dass für die Begleitpersonen auch Z-Diagnosen wie bei der familienorientierten Rehabilitation ermöglicht werden, damit diese vom Arbeitgeber frei bekommen. Dies ist ein wesentlicher Punkt, damit die Re-

\section{Hier steht eine Anzeige.}

\section{黛 Springer}


habilitationseinrichtungen auch genügend Patienten während des Jahres rekrutieren können.

4. Es ist sicherlich sinnvoll, die familienorientierte Rehabilitation auch auf andere Diagnosen auszuweiten, und es wäre möglich, diese indikationsbezogen und durch Chefärzte kontrolliert umzusetzen (z. B. dieselbe Definition für Familienorientierung: lebensbedrohliche Erkrankung, monatelanger Aufenthalt im Krankenhaus, letztlich nicht nur auf Krebserkrankungen im Kindesalter beschränkt).

5. Die Änderungen und Verbreitungen der Rehabilitationskultur sind notwendig, das Angebot wird sicherlich von vielen niedergelassenen Kolleginnen und Kollegen noch nicht ganz wahrgenommen. Es benötigt einen flachen Zugang. Es sollen bundesweit entsprechende Patienten an alle Einrichtungen zugewiesen werden.

6. Erwartungen an die Rehabilitationseinrichtungen dürfen zu Beginn nicht zu hoch gestellt werden. Ein Miteinander in der gesamten Entwicklung der Rehabilitationsszene in Österreich ist notwendig.

7. Besonders wichtig ist das Treffen mit der Kinder- und Jugendpsychiatrie und den jeweiligen Vertretern und auch der Gesellschaft, um in den Einrichtungen im Mental-healthBereich gute Konzepte zu setzen.

8. Wesentlich ist auch, das Transitionsthema zu regeln. Die Schnittstelle zum Erwachsenenalter ist gerade bei angeborenen Stoffwechselstörungen und im Mental-healthBereich, aber auch in der Hämatoonkologie wichtig. Es ist sozialversicherungsrechtlich zu klären, daß auch Auszubildende und junge Erwachsene einen Anspruch auf eine Rehabilitation in einer Kinderund Jugendlichenrehabilitationseinrichtung erhalten (Wahlrecht).

9. Wichtig ist auch, dass die Überwindung der Sprachbarriere gesichert ist und Übersetzungen möglich sind.

10. Die Qualitätssicherung der Aufgaben der Rehabilitationszentren sollten abgestimmt werden. Es wäre schön, vom Hauptverband (bzw. von der ÖGK) die vorhandene Initiative, die vom Leuwaldhof St. Veit ausgeht, zu nutzen, dass eine gemeinsame Rehabilitationsdokumentation im Kinder- und Jugendlichenbereich in Österreich aufgestellt wird. Eine Initiative bezüglich Qualitätssicherung der Rehabilitationseinrichtungen durch die Initiative Kinderrehabilitation durch Markus Wieser ist im Gange und sinnvoll.

\section{Fazit für die Praxis}

Der Anfang ist geschafft, wir können in Österreich einen eigenen hochqualitativen Weg gehen, Dank der Finanzierung und Verteilung der Betten in Österreich. Eine Reihe von Problemen sind gemeinsam mit den jeweiligen Fachspezialisten zu lösen. In Anbetracht dessen, dass wir im Vergleich zu Deutschland im Verhältnis um die Hälfte weniger Kinder- und Jugendbetten aufgestellt haben und hier nur die gewichtigeren Indikationen mit stationärem Rehabilitationsbedarf angeführt haben, gilt es jetzt, diese Betten zu nutzen, den Zugang flach zu halten und alle gemeinsam beizutragen, dieses Angebot zum Wohl der Familien, Patienten und Patientinnen zu nutzen.

\section{Korrespondenzadresse}

Prim. Univ. Prof. Dr.
Wolfgang Sperl
Zentrum für Kinder-
und Jugendmedizin
Universitätsklinik für Kinder-
und Jugendheilkunde,
Paracelsus Medizinische
Privatuniversität (PMU)
Uniklinikum Salzburg -
Landeskrankenhaus
Müllner Hauptstr. 48,
5020 Salzburg, Österreich
w.sperl@salk.at

Funding. Open access funding provided by Paracelsus Medical University.
Einhaltung ethischer Richtlinien

Interessenkonflikt. W. Sperl ist medizinischer Konsulent am Leuwaldhof, St. Veit im Pongau.

Für diesen Beitrag wurden von den Autoren keine Studien an Menschen oder Tieren durchgeführt. Für die aufgeführten Studien gelten die jeweils dort angegebenen ethischen Richtlinien.

Open Access. Dieser Artikel wird unter der Creative Commons Namensnennung 4.0 International Lizenz veröffentlicht, welche die Nutzung, Vervielfältigung, Bearbeitung, Verbreitung und Wiedergabe in jeglichem Medium und Format erlaubt, sofern Sie den/die ursprünglichen Autor(en) und die Quelle ordnungsgemäß nennen, einen Link zur Creative Commons Lizenz beifügen und angeben, ob Änderungen vorgenommen wurden.

Die in diesem Artikel enthaltenen Bilder und sonstiges Drittmaterial unterliegen ebenfalls der genannten Creative Commons Lizenz, sofern sich aus der Abbildungslegende nichts anderes ergibt. Sofern das betreffende Material nicht unter der genannten Creative Commons Lizenz steht und die betreffende Handlung nicht nach gesetzlichen Vorschriften erlaubt ist, ist für die oben aufgeführten Weiterverwendungen des Materials die Einwilligung des jeweiligen Rechteinhabers einzuholen.

Weitere Details zur Lizenz entnehmen Sie bitte der Lizenzinformation auf http://creativecommons.org/ licenses/by/4.0/deed.de.

\section{Literatur}

1. Sperl W (2017) Rehabilitation von Kindern und Jugendlichen in Österreich, Ein Blick zurück und nach vorne. Padiatr Padol 52:256-263

2. Sperl W, Nemeth C, Fülöp G, Koller I, Vavrik K, Bernert G, Kerbl R (2011) Rehabilitation für Kinder und Jugendliche in Österreich. Monatsschr Kinderheilkd 7:618-626

3. Österreichische Sozialversicherung (2018) Rehabilitationszentren für Kinder werden Realität. https://www.sozialversicherung.at/cdscontent/? contentid $=10007.846045 \&$ viewmode $=$ content . Zugegriffen: 2020

4. Bundesministerium für Soziales, Gesundheit, Pflege und Konsumentenschutz (2019) KinderReha in Österreich. https://www.gesundheit.gv. at/gesundheitsleistungen/kur-reha/kinder-reha. Zugegriffen: 2020

5. Förderverein Kinder- und Jugendlichenrehabilitation in Österreich (2020) Webpräsenz. https:// www.foerderverein-kinderreha.at/. Zugegriffen: 2020

Hinweis des Verlags. Der Verlag bleibt in Hinblick auf geografische Zuordnungen und Gebietsbezeichnungen in veröffentlichten Karten und Institutsadressen neutral. 